\title{
Stabilisation of Automated Refraction in the Immediate Time After Uneventful Cataract Surgery
}

Knud Beier Pedersen $\cdot$ Henrik Myrvold Jensen (1)

Received: May 28, 2021 / Accepted: June 28, 2021 / Published online: July 16, 2021

(c) The Author(s) 2021

\begin{abstract}
Introduction: To determine which day automated refraction stabilised the first week after uneventful cataract surgery.

Methods: This was a prospective, single-blinded, randomised cohort study of 148 eyes in 148 consecutive patients undergoing uneventful cataract surgery. Automated refraction was measured from days 2 to 7 after surgery and compared to automated refraction measured 6 weeks post-surgery. We compared measurements using a hierarchical mixed-effect model. Results: Our adjusted results did not show a statistically significant change in spherical equivalent between the first week after surgery and 6 weeks after surgery ( $p$-values: day 2: 0.914, day 3: 0.922 , day 4: 0.168 , day 5: 0.211 , day 6:
\end{abstract}

Supplementary Information The online version contains supplementary material available at https:// doi.org/10.1007/s40123-021-00372-z.

K. B. Pedersen

Eye Department, Aarhus University Hospital, Barthsgade 132 tv, 9200 Aarhus N, Denmark

H. M. Jensen ( $₫)$

Eye Department, Aalborg University Hospital, Blegdalsparken 5, 1. tv, 9000 Aalborg, Denmark e-mail: henrikdux@gmail.com

K. B. Pedersen - H. M. Jensen

Eye department of Hospital Sønderjylland, Sønderjylland, DenmarkK. B. Pedersen e-mail: knud.b.pedersen@outlook.com
0.457, day 7: 0.621). We measured the spherical error as stable except for day 5 , where a statistically significant change of 0.32 dioptre $(p=0.049)$ was detected. Similarly, the cylindrical error was also stable on all days of measurements except on day 6 , where a significant change of 0.28 dioptre $(p=0.034)$ was detected. Conclusion: The adjusted spherical equivalent was stable on days 2-7 after uneventful cataract surgery in our study population. However, as we observed a statistically significant difference in spherical and cylindrical errors on days 5 and 6 , respectively, we could not conclude that automated refraction stabilised the first week after uncomplicated cataract surgery.

Keywords: Automated refraction; Cataract; Early; Immediate; Stabilisation; Surgery; Uneventful 


\section{Key Summary Points}

\section{Why carry out this study?}

The length of time to achieve stabilisation of refraction after uneventful cataract surgery is unknown. Studies have suggested stabilisation may occur within the first week after surgery due to increasingly smaller corneal incisions.

Patient satisfaction in relation to cataract surgery will be improved if doctors can prescribe spectacles shortly after surgery minimising the time patients have without glasses.

To identify which day within the first week automated refraction stabilises after uneventful cataract surgery.

\section{What was learned from the study?}

The spherical equivalent was stable on day 2 after uneventful surgery. However, the spherical error was not stable on day 5 , and the cylindrical error was not stable on day 6.

This study could not conclude that automated refraction becomes stable during the first week after uneventful cataract surgery. Therefore, filling a spectacle prescription is not advised in the first week after surgery.

\section{INTRODUCTION}

There is a demographic trend towards a growing population of older people in the Western world and, consequently, an increase in agerelated cataract, leading to increasing demand for cataract surgery. Approximately 50,000 cataract surgeries are performed annually in Denmark [1].

There is no international consensus about the optimal timing to prescribe spectacles after cataract surgery. In Denmark, typical clinical practice suggests postponing new spectacles for at least 4 weeks, but is dependent on the clinician's discretion [2]. In the past decade, progress has been made in cataract operation techniques and equipment. Surgically induced astigmatism has been reduced using minimal corneal incisions with a size of $2.2 \mathrm{~mm}$. Also, the cumulative dissipated energy (CDE) has been reduced. $\mathrm{CDE}$ is a surrogate marker of intraoperative endothelial cell loss. An increase in CDE indicates a decreased endothelial pump function resulting in increased corneal oedema, and subsequently, a change in refraction can occur. Thus post-operative refraction may be stable earlier than previously thought using these modern surgical procedures [3].

Whilst waiting for stabilisation, patients cannot correct for astigmatism by purchasing inexpensive reading glasses. McNamara et al. reported that $60 \%$ of cataract patients in Australia were very or moderately annoyed by the lack of near-visual spectacle correction 2 weeks post-surgery, and almost $80 \%$ were annoyed 4 weeks post-surgery [4]. Therefore, reducing the post-operative waiting time to fill a spectacle prescription will promote faster visual recovery and increased patient satisfaction.

In 2018, a Danish study reported that automated refraction was stable 1 week after uneventful cataract surgery if the target refraction was not missed by more than 1.0 diopter [2]. However, a subanalysis was not completed for each specific day, and " 1 week" was defined as between 6 and 9 days in the post-operative period.

In 2013 de Juan et al. examined automated refraction post-operatively on day 1 and then weekly for a total of 4 weeks. Interestingly, their results showed a change in automated refraction between days 1 and 7 [5]. More knowledge is required about the stabilisation of refraction during the first post-operative week to identify the optimal timing for a spectacle prescription.

In Denmark, cataract operation theatres examine patients within the first week after surgery. The rationale behind this check-up is to ensure that if complications occur, such as the acute form of post-operative endophthalmitis, they can be timely diagnosed and treated [6]. If refraction stabilises post-operatively during the 
first week, a clinician could potentially offer advice on the early purchase of glasses at the mandatory first-week check-up.

This study aimed to determine which specific day, if any, automated refraction stabilised during the first week after uneventful cataract surgery.

\section{METHODS}

\section{Setting and Study Design}

All cataract surgeries were performed in the ophthalmology department of Hospital Sønderjylland. The department performs approximately 4000 cataract surgeries annually. Seven experienced cataract surgeons performed the surgeries.

This study was a prospective, cohort study comprising three visits.

1. A baseline visit 1-3 months before cataract surgery.

2. A check-up between days 2 and 7 postoperatively.

3. A post-surgical examination 6 weeks postoperatively.

At the baseline visit, patients were randomly assigned which day, post-surgery, they would receive their check-up.
Post-surgical examinations consisted of bestcorrected visual acuity (Snellen), intraocular pressure, subjective and automated refraction, slit lamp examination and an OCT scan to exclude post-operative macula oedema (see Table 1).

\section{Subjects}

Patients were included consecutively between January and December 2019. All patients underwent a baseline ophthalmological examination 1-3 months before surgery and were assessed for study inclusion.

The patients meeting the inclusion criteria (clinically relevant cataract, informed consent, referred by a general practitioner, orthoptist or an ophthalmologist outside of the hospital) were invited to participate in the study. If both eyes required surgery, the eye with the worst visual acuity was chosen for inclusion.

Exclusion criteria were: (a) significant corneal and retinal pathology that could influence refraction (b) intra- and post-operative complications. Exclusion criteria and enrolment of patients can be seen in Fig. 1 .

Randomisation for check-up day post-surgery was performed by assigning the numbers 2 to 7 to the written handouts which described the clinical trial. These numbers indicated the post-operative day each patient had to attend for a check-up examination. The numbered

Table 1 The table shows which examinations were performed at the three consultations of the study

\begin{tabular}{llll}
\hline & Baseline visit & Visit after 2-7 days & Visit after 6 weeks \\
\hline Intraocular pressure & $\mathrm{x}$ & $\mathrm{x}$ & $\mathrm{x}$ \\
Slit lamp examination & $\mathrm{x}$ & $\mathrm{x}$ & $\mathrm{x}$ \\
Indirect ophthalmoscopy & $\mathrm{x}$ & $\mathrm{x}$ & $\mathrm{x}$ \\
Automated refraction & $\mathrm{x}$ & $\mathrm{x}$ & $\mathrm{x}$ \\
BCVA & $\mathrm{x}$ & $\mathrm{x}$ & $\mathrm{x}$ \\
Lens biometry & $\mathrm{x}$ & & \\
Informed consent & $\mathrm{x}$ & $(\mathrm{x})$ & $(\mathrm{x})$ \\
(OCT + infrared photo $)$ & $(\mathrm{x})$ & &
\end{tabular}

$B C V A$ best corrected visual acuity 


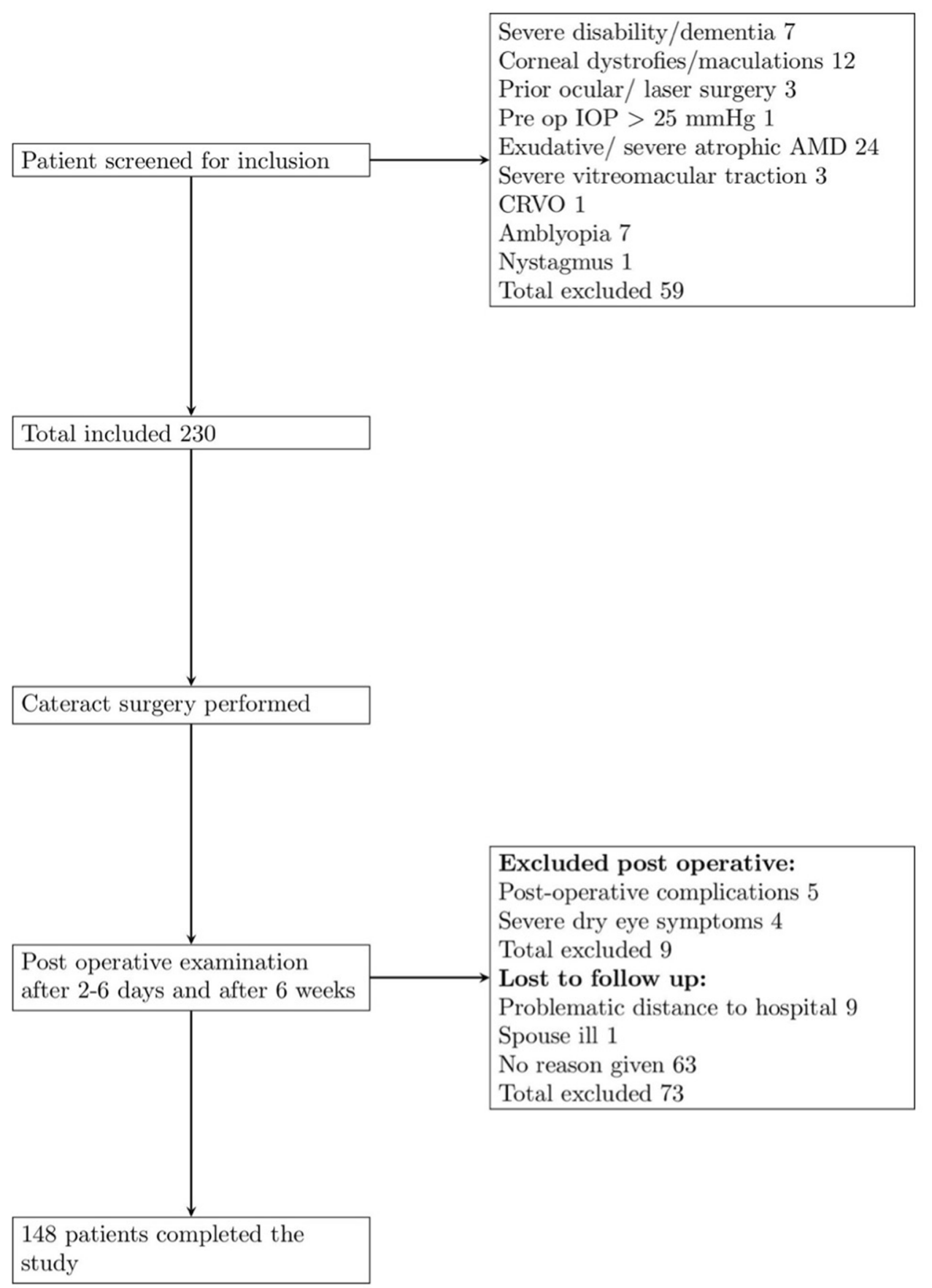

Fig. 1 Flow chart of patient enrolment

handouts were shuffled and placed in a facedown pile unaltered. At the baseline visit, the patient received the handout on the top of the pile. Afterwards, a secretary noted the handout number, and the patients were assigned to their respective post-operative check-up groups. Patients were blinded to the significance of these numbers, but the examiners were not. 


\section{Instrumentation}

All cataract surgeries were performed using the Alcon Centurion Machine (Fort Worth, TX, USA). The Nidek Tonoref II (Nidek Inc, Aichi, Japan) autorefractor and handheld refractor HandyRef-K (Nidek Inc, Aichi, Japan) were used set to RKT (refractive error/corneal curvature radius and tonometry) mode, with a range of $-30 /-20 \mathrm{D}$ to $+25 /+20 \mathrm{D}$ spherical dioptre respectively and $-12 \mathrm{D}$ cylindrical dioptre with increments of $0.25 \mathrm{D}$. All measurements included a validity score between five and nine. If the score was low, the measurement was repeated and, when necessary, background lights were dimmed, lubrication drops were administered and the eye was dilated in an attempt to increase validity. All autorefractors were calibrated and cleaned before the study.

If the target refraction (TR) was missed by more than $0.5 \mathrm{D}$ spherical equivalent, any coexisting macular oedema was examined using a spectral domain optical coherence tomography (OCT) scan (Heidelberg Spectralis HRA+OCT, Heidelberg, Germany). The Barrett intraocular lens (IOL) power calculation formula was used to calculate lens refraction, and the Alcon lens SA 60 WF was generally used. Other lenses were only used to match the fellow eye if it already had a different type of IOL implemented.

\section{Surgery}

All surgeons used a clear corneal main incision at the $11 \mathrm{o}^{\prime}$ clock position and a side port incision at the $1 \mathrm{o}^{\prime}$ clock position. The main incision measured $2.2 \mathrm{~mm}$ and surgeons used the Alcon Clear Cut S slit knife whilst the side port was made using a straight diamond knife or a $20 \mathrm{G}$ cannula. A cohesive viscoelastic solution (Provisc $^{\circledR} 0.85 \mathrm{ml}$, sodium hyaluronate $1 \%$ and occasionally DisCoVisc ${ }^{\circledR} 1 \mathrm{ml}, 4 \%$ sodium chondroitin sulfate and $1.65 \%$ sodium hyaluronate) was instilled into the anterior chamber and capsular bag to provide stabilisation. Continuous curvilinear capsulorhexis was performed with a cystotome or capsule forceps. Hydrodissection was made using a water cannula. Both horizontal chop and divide and conquer techniques were used to crack the nucleus using a Phaco Splitter.

All incisions were sealed by hydro sealing using corneal stromal saline injection, and a single absorbable suture was placed, if necessary. The CDE was recorded, and any intraoperative complications were noted. Postoperative treatment consisted of three drops daily of Spersadex (dexamethasone and chloramphenicol) and Voltabak (diclofenac) for 3 weeks.

\section{Statistical Analysis}

During the study, we completed an interim power analysis after the inclusion of 80 people. The sample size calculation used a Monte Carlo simulation, where the model was a linear regression. The power was set at $80 \%$, and the alpha level was 0.05. From this calculation, we estimated that 84 people should be included in our study. However, we chose to include 148 people to avoid overfitting when the model was fully adjusted.

All baseline variables are presented as a descriptive analysis. Continuous variables were reported with mean and standard deviation or median and interquartile range, and the categorical variables were reported with number and percentage.

An hierarchical mixed-effect model was used to estimate the fluctuation in spherical equivalents because different measurement techniques were utilised, and repeated measurements were incorporated into the study design. The covariables used to adjust the mixed-effect models to accommodate for bias included age, gender, mean preoperative $\mathrm{K} 1$ and $\mathrm{K} 2$, mean perioperative CDE (seconds) and axial length [7].

A paired $t$-test was completed as part of the sensitivity analysis to evaluate the robustness and influence of stratification on the mixed-effect models. The same analyses were completed to evaluate cylindrical and spherical error. The model control for the mixed effect models was a visual assessment of the normality of the Pearson's residuals using qq plots. A $p$-value below 0.05 or a $95 \%$ confidence interval not including 
0 was considered statistically significant. All statistical analysis utilised STATA IC 15 version 1.

The baseline data registered included age, gender, mean of preoperative $\mathrm{K} 1$ and $\mathrm{K} 2$, mean perioperative CDE (seconds), and mean axial length.

The interim sample size was calculated on the assumption that there were no different measurement techniques involved, and no stratification was required for the spherical equivalent. If this is not adjusted for in the analysis group, the three coefficients would not be significant. We deemed the sample size to have sufficient power, as the confidence intervals are narrow for this coefficient, and the coefficient is larger than the uncertainty for the machine measuring the spherical equivalent.

\section{Ethical Considerations}

Informed consent was obtained from all subjects, and the study was exempted from further ethical considerations by The Regional Committee of Ethics in Southern Denmark. All participants were treated according to the Declaration of Helsinki. Permission to store and handle data was obtained through the local data security authority. If patients refused to participate, they followed a routine post-surgical procedure.

\section{RESULTS}

A total of 148 patients met the inclusion criteria and completed the follow-up. Table 2 shows the characteristics of the participants in this study. Table 3 shows the fully adjusted estimates of the mean change of the spherical equivalent between days 2, 3, 4, 5, 6, and 7 and after 6 weeks. All changes were smaller than the uncertainty of the machine. Table 4 shows the fully adjusted estimates of the mean change of the spherical error between days 2, $34,5,6$, and 7 and after 6 weeks. On day 5 , the mean change of the spherical error was greater than the uncertainty of the machine and statistically significant. Table 5 shows the fully adjusted estimates of the mean change of the cylindrical
Table 2 Baseline data of the study population

\begin{tabular}{lll}
\hline & Level & Value \\
\hline Participants & & 148 \\
Age, mean (SD) & & $72.9(8.9)$ \\
Gender $n(\%)$ & Female & $86(58 \%)$ \\
& Male & $62(42 \%)$ \\
Axis length, mean (SD) & & $23.7(1.2)$ \\
CDE & $>20$ & $18(12 \%)$ \\
& $<20$ & $130(88 \%)$ \\
Keratometry mean (SD) & K1 & $43.203(1.4)$ \\
& K2 & $44.071(1.5)$ \\
\hline
\end{tabular}

$C D E$ cumulative dissipated energy

Some surgeons only reported whether CDE was above or below 20. For the sake of simplicity we have therefore chosen to report all CDE data in this manner

Table 3 Automated refraction: adjusted changes in spherical equivalent

\begin{tabular}{lcl}
\hline $\begin{array}{l}\text { Days after } \\
\text { surgery }\end{array}$ & $\begin{array}{l}\text { Change in spherical equivalent } \\
(\mathbf{9 5 \%} \mathbf{C I})\end{array}$ & $\begin{array}{l}\boldsymbol{p} \text { - } \\
\text { value }\end{array}$ \\
\hline 2 & $0.02(-0.33,0.37)$ & 0.914 \\
3 & $-0.02(-0.32,0.29)$ & 0.922 \\
4 & $-0.22(-0.53,0.09)$ & 0.168 \\
5 & $0.21(-0.12,0.55)$ & 0.211 \\
6 & $-0.13(-0.47,0.21)$ & 0.457 \\
7 & $-0.07(-0.35,0.21)$ & 0.621 \\
\hline
\end{tabular}

$C I$ confidence interval

error between days $2,34,5,6$, and 7 and after 6 weeks. On day 6 , the mean change of the cylindrical error was greater than the uncertainty of the machine and was statistically significant.

\section{DISCUSSION}

In our study population, the spherical equivalent was already stable on the second post- 
Table 4 Automated refraction: adjusted changes in spherical error

\begin{tabular}{lcc}
\hline $\begin{array}{l}\text { Days after } \\
\text { surgery }\end{array}$ & $\begin{array}{l}\text { Change in the spherical error } \\
(\mathbf{9 5 \% C I})\end{array}$ & $\begin{array}{l}\boldsymbol{p} \text { - } \\
\text { value }\end{array}$ \\
\hline 2 & $0.02(-0.32,0.37)$ & 0.894 \\
3 & $0.00(-0.31,0.31)$ & 0.996 \\
4 & $-0.22(-0.53,0.09)$ & 0.166 \\
5 & $0.34(0.00,0.67)$ & $0.049^{*}$ \\
6 & $0.00(-0.34,0.34)$ & 0.989 \\
7 & $-0.11(-0.39,0.16)$ & 0.421 \\
\hline CI confidence interval & \\
*indicates a statistically significant result at a level of 0.05
\end{tabular}

Table 5 Automated refraction: adjusted changes in cylindrical error

\begin{tabular}{lcl}
\hline $\begin{array}{l}\text { Days after } \\
\text { surgery }\end{array}$ & $\begin{array}{l}\text { Change in the cylindrical error } \\
(\mathbf{9 5 \% C I})\end{array}$ & $\begin{array}{l}\boldsymbol{p} \text { - } \\
\text { value }\end{array}$ \\
\hline 2 & $0.03(-0.23,0.3)$ & 0.813 \\
3 & $-0.05(-0.28,0.19)$ & 0.682 \\
4 & $0.01(-0.23,0.25)$ & 0.929 \\
5 & $-0.24(-0.5,0.02)$ & 0.066 \\
6 & $-0.28(-0.54,-0.02)$ & $0.034^{*}$ \\
7 & $0.08(-0.13,0.3)$ & 0.435 \\
\hline
\end{tabular}

$C I$ confidence interval

${ }^{*}$ indicates a statistically significant result at a level of 0.05

operative day. Previous studies have reported stable spherical equivalent after 1 week and a significant refractory shift between the first post-operative day and 1 week after surgery $[2,5,8]$. Thus, our results suggest that the spherical equivalent stabilises sooner after uneventful cataract surgery than previously reported in many patients. We also measured the change in spherical and cylindrical errors separately to unmask any potential measurement instability. These results showed that the spherical and cylindrical errors were stable on days 2-4 after surgery, but on days 5 and 6 a small, but statistically significant, change in spherical and cylindrical errors was observed when compared to 6 weeks after surgery. Thus, we could not conclude that the spherical and cylindrical errors were stable throughout the entire first week, although there appeared to be a trend towards stability. In comparison, de Juan et al. reported that the spherical and cylindrical errors were stable 1 week after surgery compared to 4 weeks. In contrast, Ostri et al. reported that the cylindrical error changed significantly between weeks 1 and 4 . In addition, Caglar et al. found a significant change in spherical error between 1 and 4 weeks. These studies support our results indicating that spherical and cylindrical errors may not be stable the first week post-surgery. Before conducting our study, we hypothesised that any observable change in refraction in the first week would, most likely, be greatest the first few days post-surgery due to immediate post-operative sequelae. The reason the significant change occurred relatively late in the week (on days 5 and 6) is unclear. We noted that these changes, though statistically significant, are only slightly larger than the intrinsic measuring uncertainty of the machine. However, since even small refraction changes can substantially influence visual acuity and subjective perception of vision, most optometrists would be hesitant to prescribe spectacles earlier than current guidelines suggest based on our results.

\section{Strengths}

In this study, cataract surgery was performed by seven different surgeons with varying degrees of experience. This variation is a strength of the study as it improves the external validity. The second strength of our study was the population. We can safely assume that these patients represent nearly all patients requiring cataract surgery as there were no private eye clinics offering cataract surgery at the time of the study. Finally, the use of the hierarchical mixedeffect model has several advantages. The power of this type of model is high because of repeated measurements and its ability to model the variance and include multiple strong predictors 
so that the bias in the regression coefficients are minimised [7].

\section{Limitations}

The results are only valid for patients fulfilling the inclusion and exclusion criteria. We did not measure the central corneal thickness to assess post-op corneal swelling and thus cannot exclude the potential effect on refraction. However, de Juan et al. reported that although corneal swelling continued to significantly decrease 2 weeks post-surgery, it did not influence postoperative automatic refraction after 1 week. There was a substantial number of patients lostto-follow-up, considering the size of our study population. It is reasonable to assume that a study participant experiencing post-operative discomfort (dry eyes, pain and blurring) would more likely be compliant with follow-up. In addition, discomfort resulting from dry eyes could influence the accuracy of automated refraction. However, our results show relatively stable refraction in the first week after surgery. Therefore, it is debatable whether loss to followup increases the risk of biased results. Lastly, at the baseline consultation, it was not possible in the clinical setting to blind the examiners to the post-operative day the patients were assigned for check-up. This, in theory, could open the possibility for selection bias.

Future studies could use corneal topography to assess the stability of the cylindrical axis and compare visual acuity with results from the purchased spectacles to establish if it would be advisable to prescribe spectacles earlier than current guidelines suggest.

\section{CONCLUSION}

In this study, the spherical equivalent was stable from days 2 to 7 after uneventful surgery, which was also observed for spherical and cylindrical errors except on days 5 and 6 . Based on these results, we could not conclude that automated refraction had stabilised within the first week post- surgery. We suggest that future studies should further elucidate this subject.

\section{ACKNOWLEDGEMENTS}

We thank the participants of the study.

Funding. Funding for the study was exclusively provided by the department of ophthalmology of the University Hospital of Southern Denmark. The same department also covered the Rapid Service Fee.

Medical Writing and Editorial Assistance. The authors would like to thank Rune Holmbjoern, chief physician of the department of ophthalmology of the University Hospital of Southern Denmark, for providing facilities and financial support along with mentorship. Also, many thanks should be extended to Christian Backer Mogensen, clinical professor at the Department of Regional Health Research, University of Southern Denmark for timely critique and constructive advice. Finally, we would like to thank statistician Andreas Pedersen, also from the Department of Regional Health Research, University of Southern Denmark, for assistance with all statistical models and calculations.

Authorship. All named authors meet the International Committee of Medical Journal Editors (ICMJE) criteria for authorship for this article, take responsibility for the integrity of the work as a whole, and have given their approval for this version to be published.

Authorship Contributions. Dr. Knud B. Pedersen and Dr. Henrik M. Jensen contributed equally to this work as first authors.

Disclosures. Dr. Henrik Myvold Jensen was affiliated with the department of ophthalmology of the University Hospital of Southern Denmark, Sønderborg at the time of the completion of this study. At the time of publication of this study, he is affiliated with the department of ophthalmology at Aalborg University Hospital, Denmark. Dr. Knud Beier Pedersen was affiliated with the department of ophthalmology of the University Hospital of Southern Denmark, Sønderborg at the time of the completion of this study. At the time of publication 
of this study, he is affiliated with the department of ophthalmology at Aarhus University Hospital, Denmark.

Compliance with Ethics Guidelines. This study was conducted in accordance with the declaration of Helsinki and it was exempted from further ethical considerations by The Regional Committee of Ethics in Southern Denmark.

Data Availability. All data generated or analyzed during this study are included in this published article/as supplementary information files.

Open Access. This article is licensed under a Creative Commons Attribution-NonCommercial 4.0 International License, which permits any non-commercial use, sharing, adaptation, distribution and reproduction in any medium or format, as long as you give appropriate credit to the original author(s) and the source, provide a link to the Creative Commons licence, and indicate if changes were made. The images or other third party material in this article are included in the article's Creative Commons licence, unless indicated otherwise in a credit line to the material. If material is not included in the article's Creative Commons licence and your intended use is not permitted by statutory regulation or exceeds the permitted use, you will need to obtain permission directly from the copyright holder. To view a copy of this licence, visit http://creativecommons.org/licenses/by$\mathrm{nc} / 4.0 /$.

\section{REFERENCES}

1. Andersen KL. Behandling af aldersbetinget grå stær https://www.sst.dk/: Danish Health Authority; 2014. https://www.sst.dk/da/nyheder/2014/behandling-afaldersbetinget-graa-staer. Accessed 27 April 2021.

2. Ostri C, Holfort SK, Fich MS, Riise P. Automated refraction is stable 1 week after uncomplicated cataract surgery. Acta Ophthalmol. 2018;96(2): 149-53.

3. Chun AG. Effects of cumulative dissipated energy on postoperative corneal pachymetry in resident performed cataract surgery. Investig Ophthalmol Vis Sci. 2012;53(14):6729.

4. McNamara P, Hutchinson I, Thornell E, Batterham M, Iloski V, Agarwal S. Refractive stability following uncomplicated cataract surgery. Clin Exp Optom. 2019;102(2):154-9.

5. de Juan V, Herreras JM, Pérez I, Morejón Á, RíoCristóbal A, Martín R, et al. Refractive stabilization and corneal swelling after cataract surgery. Optomet Vis Sci. 2013;90(1):31-6.

6. Durand ML. Endophthalmitis. Clin Microbiol Infect. 2013;19(3):227-34.

7. Gail MH, Wieand S, Piantadosi S. biased estimates of treatment effect in randomized experiments with nonlinear regressions and omitted covariates. Biometrika. 1984;71(3):431-44.

8. Caglar C, Batur M, Eser E, Demir H, Yaşar T. The stabilization time of ocular measurements after cataract surgery. Semin Ophthalmol. 2017;32(4): 412-7. 